\title{
Surgical management of ocular surface disorders using conjunctival and stem cell allografts
}

\author{
Douglas J Coster, Rajesh K Aggarwal, Keryn A Williams
}

\begin{abstract}
Aims-The aim of this work was to investigate different surgical options for the repair of the ocular surface, using various extensions of the procedure of limbal stem cell allotransplantation.

Methods/Results-Straightforward lamellar limbal transplantation was performed in one patient with contact lens induced limbal stem cell failure. A second patient with a neoplastic corneal lesion underwent limbal allotransplantation, followed later by a second procedure in which $360^{\circ}$ of limbus and the entire ocular surface was transplanted. A third patient who had suffered extensive chemical burns was treated by penetrating keratoplasty to restore central corneal clarity, followed later by a lamellar allograft comprising a $360^{\circ}$ annulus of peripheral cornea to repair the ocular surface. A fourth patient with long standing, chronic trachomatous eye disease underwent allotransplantation of the upper lid tarsal plate and conjunctiva, with reconstruction of the fornix. Finally, a child with Goldenhar's syndrome underwent reconstruction of the medial fornix with autologous buccal mucosa, followed by a lamellar corneal and conjunctival allograft. A stable ocular surface has been achieved in each case and there have been no obvious rejection episodes.

Conclusion-Limbal allotransplantation can be extended to engraftment of the entire superficial cornea, limbus, conjunctiva, and tarsal plate in patients with a range of pathologies. We have described the surgical management of five cases which demonstrate the potential of the technique, but which raise questions which still need to be explored.

(Br f Ophthalmol 1995; 79: 977-982)
\end{abstract}

Ocular surface disorders affecting the cornea can be very damaging to vision. The corneal surface is a critical component of the optical system of the eye. For the corneal surface to fulfil its optical function, it must retain a normal ultrastructure which depends on maintaining normal epithelial differentiation. Disorders of the ocular surface are common owing to the exposed nature of the external eye and the way in which the mucosal surface responds to challenge. The sequelae of inflammation disturb the ultrastructure of the cornea, and may affect the differentiation of the surface epithelium. Ocular surface disorders may result from pathology in the surface epithelium, the tear film, or the eyelids.
The corneal epithelium is regenerated from stem cells situated at the limbus. ${ }^{12}$ These cells are susceptible not only to noxious external stimuli, such as chemicals and invading pathogens, but also to endogenous inflammatory and cicatrising diseases, such as ocular pemphigoid, Stevens-Johnson syndrome, and trachoma. As a result of these processes the ocular surface can be irreparably damaged, with serious consequences both for vision and ocular comfort.

Under these circumstances, it has been tempting to offer corneal transplantation, but graft failure is almost invariable in cases where there is marked ocular surface disease. Epithelial instability, persistent epithelial defects, and subsequent vascularisation are the usual precedents of graft failure. These changes in the epithelium of the cornea have recently been attributed to limbal stem cell failure. In consequence there has also been increasing interest in managing these conditions with limbal grafts. ${ }^{3-5}$

The effective use of limbal stem cell grafts demands an understanding of the pathology and clinical manifestation of stem cell disease, the surgical technique required, and the implications of using such grafts. Conjunctival ${ }^{3}$ and limbal ${ }^{45}$ autografts have previously been described, but these approaches have at least two important limitations: the lack of normal autologous limbus and the extensive conjunctival disease in some patients.

Our interest in the use of conjunctival and limbal allografts arose when we found it necessary to transplant limbal cornea and conjunctiva for patients with stem cell disease where there was no normal autogenous donor tissue available. Surprisingly, we observed that the conjunctiva did not appear to reject as anticipated, although we have observed transient vascular changes and oedema which may have been the clinical manifestation of allograft reactions. We have subsequently expanded the scope of conjunctival transplantation and incorporated related tissues, such as cornea, limbus, tarsal cartilage, and conjunctival fornix into the grafts as required. In this paper, we report five cases which demonstrate the potential of this approach.

\section{Methods and results}

\section{CASE REPORTS}

Case 1

A 43-year-old woman first presented in 1983. She was myopic (approximately $-2.00 \mathrm{D}$ ) and, until about 6 months before presentation, had 
been wearing daily wear soft contact lenses quite successfully for 10 years. At presentation, her eyes had become red and uncomfortable and her best corrected vision had reduced to $6 / 12$. This level of vision was attributable to corneal pathology. Both corneas were affected by superficial vascularisation with vortex epithelial opacity around the edges of limbal vascular ingrowths. Biopsy revealed 'epithelial dysplasia'. The patient was returned to the care of her ophthalmologist with a provisional diagnosis of contact lens induced corneal epithelial dysplasia. As advised, she discontinued contact lens wear.

She was not seen again for 8 years. In 1991, her symptoms had progressed and she was now disabled by reduced vision and chronic pain and photophobia. Her visual acuity was right $6 / 24$ and left $6 / 60$. The corneal pathology was more advanced, with extensive superficial corneal vascularisation involving the entire corneal periphery. The corneal epithelium was opaque, with an obvious vortex pattern. The external ocular surface, including the conjunctiva, the lids, and tear film were all normal.

A diagnosis of 'contact lens induced epithelial stem cell failure' was postulated and corneal limbal transplantation was proposed for the most affected eye. An allograft was necessary because there was no normal limbal tissue from which an autograft could be taken.

The patient received two left limbal allografts from a donor eye in 1992. Thin lamellar dissections were performed in order to create beds into which the grafts could be laid. Areas comprising $2 \mathrm{~mm}$ wide sections of the peripheral cornea and limbus with $5 \mathrm{~mm}$ fringes of conjunctiva were excised over 4 clock hours at 10-2 o'clock and 4-8 o'clock. Templates of the defects were made in order to facilitate excision of precisely matching grafts from the donor eye. The lamellar grafts needed to be thick enough to bear the undisturbed epithelium and to be able to be sutured into the recipient cornea so that the donor and recipient corneal surfaces were flush with each other. Systemic immunosuppression was employed. The patient received systemic azathioprine (maintenance dose of $1 \mathrm{mg} / \mathrm{kg} /$ day) and cyclosporin A (to maintain serum levels between $310-500 \mathrm{ng} / \mathrm{ml}$ ), commencing 5 days before surgery.

Within days of the surgery, the corneal surface began to improve. It regained the normal appearance of corneal epithelium. Vascularisation regressed over a period of months. Corresponding with the improvement in the cornea, the visual acuity improved. Within 3 months, acuity had improved to $6 / 12$ in the grafted eye and to a lesser extent $(6 / 18)$ in the non-grafted eye, with her old spectacle correction. At no stage was there any evidence of allograft rejection. Thirteen months later, she had developed hirsutism and the systemic immunosuppression was stopped. Within 6 weeks, both eyes deteriorated and symptoms of photophobia, ocular discomfort, and reduced visual acuity returned.

Slit-lamp examination showed progression of the disease in the right eye, with increased epithelial punctate keratopathy with corneal oedema and a whorl-like pattern to the epithelium. The grafted eye showed an irregular epithelium with superficial epithelial lines akin to those seen in allograft rejection of corneal buttons. Visual acuity dropped to right $6 / 36$ and left $6 / 18$.

Systemic immunosuppression was started again, as previously, and after a week symptoms started to settle. One month later, the left eye stabilised with a visual acuity of $6 / 12$, but the right continued to be troublesome and has recently undergone a conjunctival and corneal limbal allograft. The left eye remains stable 29 months after surgery but the patient does seem to require systemic immunosuppression.

\section{Case 2}

A 65-year-old man presented with an extensive neoplastic lesion of the limbus of the left cornea. The lesion involved almost $270^{\circ}$ of the limbus. An excision biopsy was performed and histology was reported as showing a 'carcinoma in situ of the corneal limbus' with complete excision. However, within 4 months of surgery, the lesion reappeared in the area of previous excision and in the apparently normal limbus which had been left at the time of the original surgery (Fig 1A). A second excision was performed and cryoablation of the limbus was carried out at the time of the surgery.

The man subsequently remained free of tumour recurrence, but his vision deteriorated. The cornea developed opaque epithelium, which stained with Bengal Rose revealing a vortex pattern. There was a mild degree of superficial corneal oedema (not enough to alter pachymetry) and extensive corneal vascularisation. The patient was in constant discomfort, and his vision was reduced to $6 / 60$. It was considered that he had developed changes in the corneal epithelium as a result of surgery and cryoablation of the limbus, with subsequent stem cell failure.

A limbal graft was suggested with the aim of improving vision and stabilising the corneal surface. The patient was not prepared to have the normal contralateral eye used as a source of tissue. He was prepared to have an allograft but did not wish to have systemic immunosuppression. Limbal stem cell grafts were performed as described above. Postoperatively, the patient received topical prednisolone phosphate $0.5 \%$ only.

The corneal surface changed within a few days of surgery and the process of improvement continued for several months (Fig 1B). Within 3 months, vision was $6 / 18$. The corneal surface appeared normal, there was no staining with Bengal Rose and corneal vascularisation regressed. The clinical appearance remained unchanged for 12 months, but then regressed with surface changes and vascularisation (Fig 1C).

A second procedure involving allotransplantation of $360^{\circ}$ of limbus and the entire corneal surface was then performed. The whole of the recipient's superficial cornea was excised to a depth of $25 \%$ and $5 \mathrm{~mm}$ of surrounding 


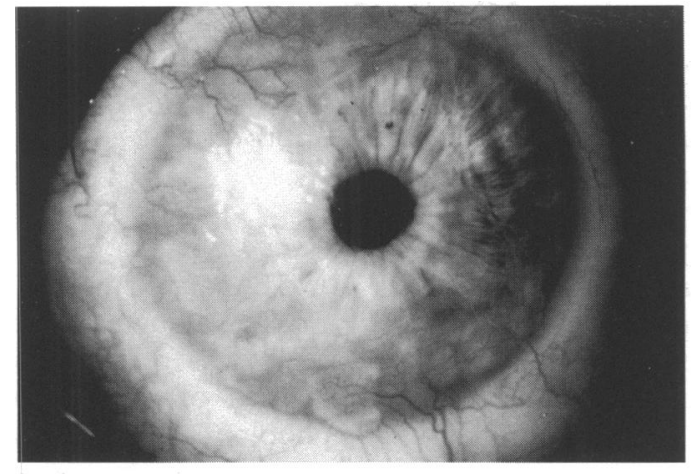

Fig $1 A$

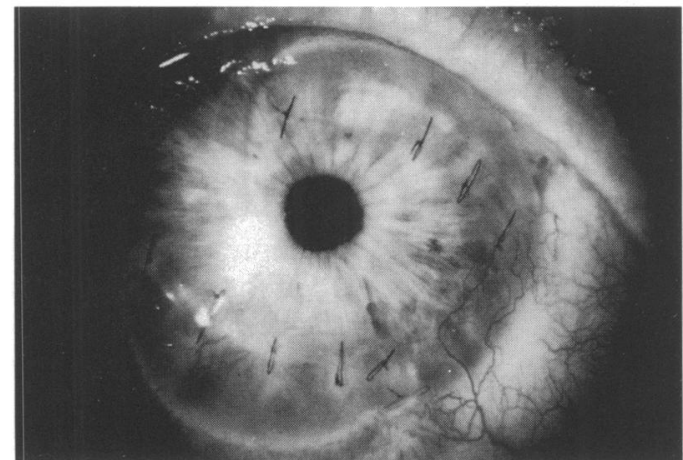

Fig $1 B$

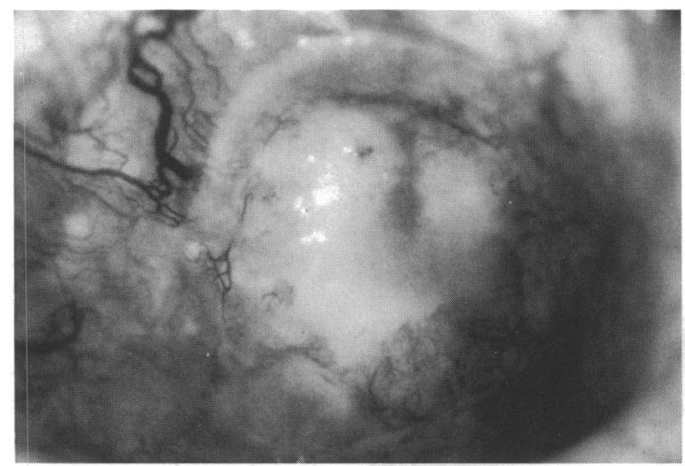

Fig $1 C$

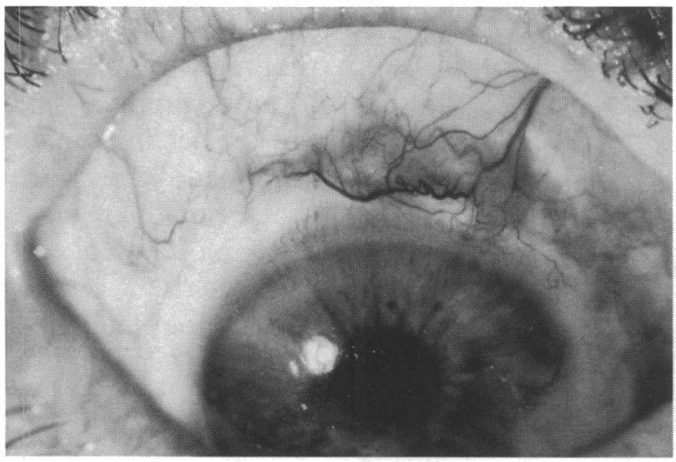

Fig $1 D$

Figure 1 Case 2. (A) Recurrent intraepithelial carcinoma. These lesions had recurred after previous excision and cryoablation. (B) The ocular surface was stable for 6 months after limbal transplantation. (C) After 6 months of epithelial stability, the patient developed recurrent epithelial disease with dysplasia and vascularisation which eventually progressed to epithelial instability and ulceration. (D) After a second procedure, in which the entire corneal surface was transplanted, along with the limbus and $10 \mathrm{~mm}$ of conjunctiva, the ocular surface remained stable and the vision improved to $6 / 18$. conjunctiva was also removed. A graft of corresponding size was taken from a donor eye and secured over the recipient cornea with four deep 10-0 nylon sutures at the corneoscleral junction and 12 8-0 Vicryl sutures at the conjunctival edge. Postoperatively, the patient received topical prednisolone phosphate $0.5 \%$ as before. This procedure was successful and the patient's ocular surface has remained stable for 8 months after surgery (Fig 1D).

\section{Case 3}

A 36-year-old man was attacked by an assailant who poured concentrated acid over his head and neck. He sustained extensive burns and chemical injury to both eyes. His vision was light perception from the time of the assault, with severe corneal damage and cataracts (Fig 2A). The cataracts were removed elsewhere within a few months of the injury, but his vision remained poor. $\mathrm{He}$ was later referred for consideration of corneal transplantation and reconstructive plastic surgery for his facial scars.

Various plastic surgery procedures were carried out before any attempt was made to improve his vision. At this stage, he was barely able to perceive light, although he had accurate projection. The cornea, conjunctiva, and eyelids were severely scarred. The ocular surface was also extensively keratinised and was so abnormal that it was impossible to determine accurately the intraocular pressure, although it was believed to be $18-30 \mathrm{~mm} \mathrm{Hg}$ on most occasions.

The patient was a highly intelligent professional engineer, and he and his family were determined to pursue any course which offered even a faint hope of restoring his vision. Under these circumstances, a right corneal graft was performed under cover of systemic immunosuppression (azathioprine maintenance dose of $1 \mathrm{mg} / \mathrm{kg} /$ day; cyclosporin A to maintain serum levels between $310-500 \mathrm{ng} / \mathrm{ml}$ ).

The graft functioned well initially, providing the patient with navigational vision. Although the intraocular pressure was less than $25 \mathrm{~mm} \mathrm{Hg}$ after surgery, the optic disc was grossly cupped. After 6 months, the useful vision which had been achieved began to fail because of epithelial instability. Epithelial defects developed which tended to heal slowly (Fig 2B). The epithelium became opaque and a vortex pattern of epithelial erosions was apparent after staining with Bengal Rose. Eventually, vision was reduced to the perception of light and it became difficult to see into the eye beyond the epithelium. The graft did not thicken appreciably: pathology was confined to the epithelium. Although this might have been allograft rejection, the clinical opinion was that ocular surface disease was the main problem and that limbal stem cell failure as a consequence of the chemical burn was contributing to the pathology of the corneal epithelium.

A circumferential limbal allograft was performed. The penetrating graft was left untouched, but a $360^{\circ}$ ring of peripheral host 
cornea was excised to a depth of approximately $25 \%$, extending from the limbus to the edge of the central penetrating graft. A corresponding annulus of peripheral superficial cornea, limbus and a $10 \mathrm{~mm}$ conjunctival fringe was removed from a donor eye. The graft was secured centrally to the penetrating graft with 12 10-0 nylon sutures and the conjunctiva was sutured to the host conjunctiva with $128-0$ Vicryl sutures. The systemic immunosuppression with azathioprine and cyclosporin A that had been commenced at the time of the initial corneal graft was continued.

Over the next 6 weeks, the surface of the cornea improved considerably. Epithelial stability was achieved and maintained (Fig 2C). Vision improved and it became possible to see through the cornea to examine the posterior segment of the eye. Unfortunately, the optic nerve morphology had further deteriorated, despite a reasonable intraocular pressure. The

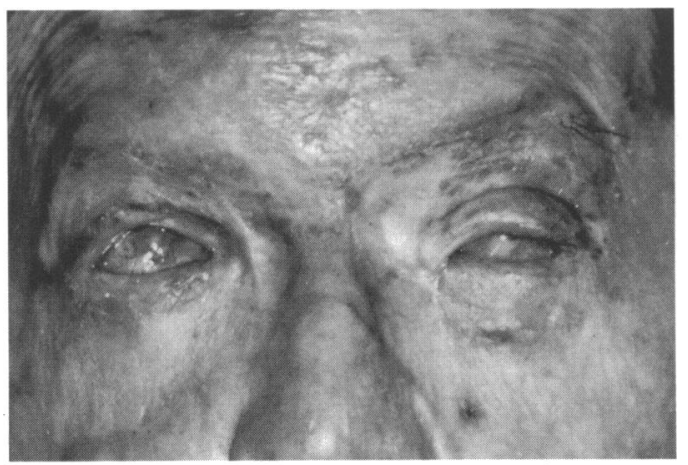

Fig $2 A$

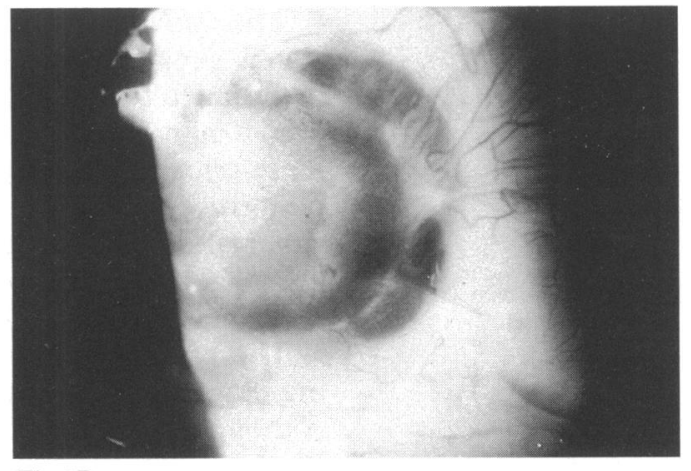

Fig $2 B$

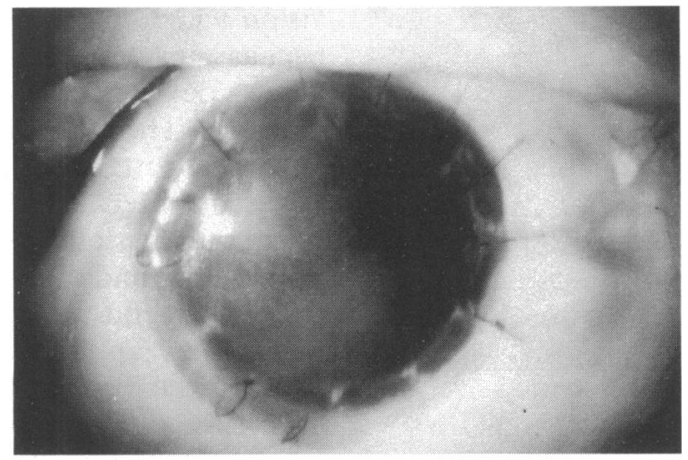

Fig 2C

Figure 2 Case 3. (A) A 36-year-old man suffered bilateral severe acid burns as a result of an assault. There was corneal opacification and extensive and severe disruption of the ocular surface. (B) $A$ penetrating corneal graft developed recurrent epithelial ulceration but escaped allograft rejection. (C) After allotransplantation of limbus and conjunctiva the ocular surface was re-stabilised and maintained. patient did, however, retain sufficient vision for elementary navigation. Twelve months after surgery, the ocular surface has maintained its improvement.

\section{Case 4}

A 57-year-old man with a history of chronic trachomatous eye disease since the age of 20 years was referred to us in 1989 . He complained of severe ocular discomfort, photophobia, and reduced visual acuity. On examination, his visual acuities were right $3 / 36$ and left $6 / 24$. He showed bilateral changes of chronic trachoma with severe scarring and keratinisation of the tarsal plates, but surprisingly little entropion or trichiasis. He had bilateral corneal vascularisation, anterior stromal scarring, and unhealthy corneal epithelium. He was initially treated with upper tarsal scraping to remove plaques of keratin and topical vitamin A ointment $0.05 \%$. Clinically, there was a significant improvement, the eyes becoming comfortable and visual acuity improving to right $6 / 24$ and left $6 / 18$.

The patient subsequently defaulted from follow up until 1993, when he returned with a painful left eye, gross keratinisation and scarring of the upper tarsal plate, corneal vascularisation, and an unhealthy epithelium with multiple defects. A further trial of vitamin $A$ ointment $0.05 \%$ failed to relieve his symptoms.

Three months later he underwent a left upper lid tarsal allograft. The patient's tarsal plate was excised together with the overlying conjunctiva. Care was taken to leave the lid margin intact. The entire upper fornix was removed with the bulbar conjunctiva, as far as the limbus. A corresponding excision was made from a block of donor tissue which included the upper eyelid and the whole globe. Permission for this extended harvest of tissue from the donor was specifically obtained from the donor's relatives. The donor tarsal plate with conjunctiva was inlaid into the surgically created defect in the recipient. Both tarsal plate and conjunctiva were secured with 8-0 Vicryl sutures. Postoperatively, he received topical prednisolone phosphate $0.5 \%$ but no systemic immunosuppression. His symptoms improved: the eye became quieter, corneal vascularisation regressed, the corneal epithelium became much healthier, and visual acuity stabilised at $6 / 12$. No clinical episode of allograft rejection was noted.

Histology of the excised tissue suggested squamous metaplasia and scarring consistent with old trachoma, but a well differentiated squamous cell carcinoma could not be ruled out. In early 1994, the patient developed an atypical area with gross keratinisation at the graft-host interface. This was excised and histology confirmed it to be well differentiated squamous cell carcinoma.

The graft itself remains relatively healthy and the patient is more comfortable than for many years, with visual acuity of right $6 / 24$ and left $6 / 18$, and with no sign of recurrence after 9 months. The patient is well aware of the 
diagnosis but is at present reluctant to undergo any further surgery.

Case 5

A 3-year-old boy with Goldenhar's syndrome presented in May 1988. He had an unusual association of a right upper lid coloboma with adherence of the medial fragment of the eyelid to the full thickness corneal dermoid, and loss of the associated fornix. Visual acuity was reduced to perception of light in the affected eye and the parents' main concern was the cosmetic appearance of this eye. The other eye was normal. Surgical correction was undertaken. The upper medial portion of the lid was dissected off the cornea and the upper lid defect primarily closed. The upper medial fornix was reconstructed using mucous membrane collected from inside the lower lip and a bandage contact lens was inserted to maintain the fornix.

Over the next 2 years, the boy developed marked superonasal symblepharon and further growth of the corneal dermoid, preventing the use of a cosmetic contact lens. At the age of 9 years he underwent a lamellar corneal and conjunctival allograft to restore the regularity of the cornea and re-fashion the upper fornix. An area of superficial cornea was excised. The edges were first marked with a $5 \mathrm{~mm}$ trephine to create a well defined area and the attached conjunctival symblepharon was removed along with the superficial cornea. A template was used to prepare a graft of the correct size from a donor eye. The corneal edge of the graft was secured with 10-0 nylon and the conjunctival edges were sutured with 8-0 Vicryl. No systemic immunosuppression was used but topical prednisolone phosphate $0.5 \%$ was administered four times a day.

Seven months after surgery, the boy maintains a smooth corneal surface and deep fornices, allowing comfortable wear of a cosmetic contact lens. To date, no clinical allograft rejection episode has been recorded.

\section{Discussion}

A wide range of ocular surface disorders may be amenable to reconstruction with various allografts. Corneas affected by epithelial diseases affecting the limbus develop linear and punctate epithelial opacities, recurrent epithelial defects, stromal oedema and vascularisation, and keratinisation of the ocular surface. Limbal disease may result in a disturbance of corneal disease incompatible with good vision (as in cases $1,2,3$ ). If the ocular surface is abnormal, corneal transplantation may be precluded (case 3 ). The conjunctiva may be relatively unaffected but in some diseases the cornea, limbus, and conjunctiva may all be abnormal, as is often the case in trachoma (case 4). In other conditions, the conjunctiva may be deficient to such an extent that the mobility and position of the lids are grossly disturbed (case 5).

There has been a long history of surgical procedures developed with the aim of restoring the morphology of the ocular surface. ${ }^{6-9}$ Most of these procedures have been based on the use of autografts of skin and mucous membranes collected from various sites. All of these procedures fail to provide appropriately differentiated epithelium, resulting in an inappropriate corneal surface with important dysfunctional consequences.

Limbal autograft transplantation is the preferred surgical technique for treating ocular surface disorders. It is usually safe to collect the donor limbus from a normal limbus in the contralateral eye. This is possible when the disease is unilateral, as is usually the case with tumours, and sometimes with chemical burns. However, it is important to be sure that the limbus in the donor eye is normal, and not exhibiting mild pathology. Failure to notice corneal disease can result in a severe decrease of vision in the donor eye. ${ }^{10}$ Furthermore, not all patients are prepared to risk compromising their better eye. In these cases, and where there is extensive bilateral disease, it becomes necessary to consider other alternatives, including the use of allografts. ${ }^{11} 12$

Although the results of limbal allotransplantation have been encouraging in some instances, many issues have yet to be resolved. These include establishment of the most appropriate surgical technique, the best method for storage of donor tissue, and the long term fate of the grafts. The surgical techniques are still evolving. In all the cases described, corneal neovascularisation was superficial rather than deep, and no attempt was made to obliterate the vessels at surgery. However, in each case regression of surface corneal vessels was observed after surgery. It would seem from case 2 that larger limbal grafts fare better than smaller grafts: two small grafts did not do as well as a subsequent graft in which the whole limbus was transplanted. Many patients with ocular surface disorders have extensive pathology, with gross conjunctival shrinkage as well as limbal abnormalities. The amount of conjunctiva that needs to be included with the grafted tissue is uncertain. Management of the donor material, which may include conjunctiva and lid tissue, is another matter to be considered. We have adopted a policy of using such tissue within 24 hours of collection, but the precise limits of storage have not been determined.

The fate of limbal and conjunctival allografts, in these circumstances, is unclear. One would expect the grafts to be rejected but this does not invariably happen, as is illustrated by the above cases. Allografts may survive in the absence of immunosuppression, but the prognosis improves in patients in whom systemic immunosuppression is justifiable. If allograft rejection does occur it does so in an unexpected form. The histology of conjunctiva is similar to skin. Certainly, nothing resembling the rejection of skin allografts was seen in these patients. The circulation in the conjunctival segment of the allograft is re-established within a few days of transplantation, but a period of vascular stasis and oedema usually occurs between 4-14 days after surgery. The phase is transient, lasting 3-4 days, after which the grafted 
conjunctiva regains a normal appearance and functions accordingly. Whether this conjunctival oedema reflects rejection is unknown. Such a phase was observed in the second graft in case 1: analysis of the DNA genotype of cells harvested by impression cytology from the central cornea of this patient at 20 weeks postoperatively showed no detectable donor cells, perhaps suggestive of rejection, yet the clinical improvement at this time was substantial and continues to be maintained. ${ }^{13}$ Limbal allograft failure possibly caused by rejection has been reported by others. ${ }^{14}$

The tantalising early success of limbal stem cell allografting poses many questions which will need to be answered if the technique is to advance and become a routine clinical procedure. What is the fate of allografted limbal tissue? Does allograft rejection occur but go undetected? Is immunosuppression required, and if so, how much and for how long? How is the graft tissue best preserved? The procedure has been remarkably effective in a small number of cases. The use of conjunctival and tarsal plate allografts to reform the fornix expands the range of diseases for which this form of treatment may be an option. However, more procedures need to be carried out and followed if the potential of the procedure is to be explored, and there is need for laboratory studies to explore the underlying biological processes.

1 Schermer A, Galvin S, Sun T-T. Differentiation-related expression of a major $64 \mathrm{~K}$ corneal keratin in vivo and in culture suggests limbal location of corneal epithelial stem cells. F Cell Biol 1986; 103: 49-62.

2 Thoft RA, Wiley LA, Sundarraj N. The multipotential cells of the limbus. Eye 1989; 3: 109-13.

3 Thoft RA. Conjunctival transplantation. Arch Ophthalmol 1977; 95: 1425-7.

4 Kenyon KR, Tseng SCG. Limbal autograft transplantation for ocular surface disorders. Ophthalmology 1989; 96: 709-23

5 Tseng SCG. Concept and application of limbal stem cells Eye 1989; 3: 141-57.

6 Leone CRJ. Mucous membrane grafting for cicatricial entropion. Ophthalmic Surg 1974; 5: 24-8.

7 Levin PS, Dutton J. Polytef (polytetrafluoroethylene) alloplastic grafting as a substitute for mucous membrane. Arch Ophthalmol 1990; 108: 282-5.

8 Naumann GOH, Lang GK, Rummelt V, Wigand ME. Autologous nasal mucosa transplantation in severe Autologous nasal mucosa transplantation in severe Ophthalmology 1990; 97: 1011-7.

9 Shore JW, Foster CS, Westfall CT, Rubin PAD. Results of buccal mucosal grafting for patients with medically controlled ocular cicatricial pemphigoid. Ophthalmology 1992 99: 383-95.

10 Jenkins C, Tuft S, Liu C, Buckley R. Limbal transplantation in the management of chronic contact lens associated epitheliopathy. Eye 1993; 7: 629-33.

11 Pfister RR. Corneal stem cell disease: concepts, categorization, and treatment by auto and homotransplantation of limbal stem cells. CLAO f 1994; 20: 64-72.

plantation of limbal stem cells. CLAO f 1994; 20: 64-72.
12 Tsai RJF, Tseng SCG. Human allograft limbal transplantation for corneal surface reconstruction. Cornea 1994; 13: tion for com

13 Williams KA, Brereton HM, Aggarwal R, Sykes PJ, Turner DR, Russ GR, et al. Use of DNA polymorphisms and the polymerase chain reaction to examine the survival of human limbal stem cell allograft. Am $\mathcal{f}$ Ophthalmol (in press).

14 Thoft RA, Sugar J. Graft failure in keratoepithelioplasty. 